Article

\title{
Fractal Structure of Hadrons: Experimental and Theoretical Signatures
}

\author{
Airton Deppman ${ }^{(1)}$ \\ Instituto de Física-Universidade de São Paulo, São Paulo 05508-090, Brazil; deppman@if.usp.br \\ Received: 28 June 2017; Accepted: 14 August 2017; Published: 26 August 2017
}

\begin{abstract}
One important ingredient in the study of cosmological evolution is the equation of state of the primordial matter formed in the first stages of the Universe. It is believed that the first matter produced was of hadronic nature, probably the quark-gluon plasma which has been studied in high-energy collisions. There are several experimental indications of self-similarity in hadronic systems-in particular in multiparticle production at high energies. Theoretically, this property was associated with the dynamics of particle production, but it is also possible to relate self-similarity to the hadron structure-in particular to a fractal structure of this system. In doing so, it is found that the thermodynamics of hadron systems at equilibrium must present specific properties that are indeed supported by data. In particular, the well-known self-consistence principle proposed by Hagedorn 50 years ago is shown to be valid, and can correctly describe experimental distributions, mass spectrum of observed particles, and other properties of the hadronic matter. In the present work, a review of the theoretical developments related to the thermodynamical properties of hadronic matter and its applications in other fields is presented.
\end{abstract}

Keywords: thermodynamical properties; hadronic matter; high energy collisions

PACS: 12.38.Mh; 13.60.Hb; 24.85.+p; 25.75.Ag

\section{Introduction}

One of the most striking features of high-energy collisions (HECs) is the formation of a system in thermodyanmical equilibrium which subsequently decays through the emission of several particles. In spite of the fact that evidence of the existence of such a system-which received the name "fireball" — was already present in the 1950s [1], the idea of fireballs was somewhat challenged by experimental data as the collision energy increased. Today there is no doubt that the outcomes of HEC is mostly determined by the decay of a fireball, but the complete thermodynamical description is still controversial.

The existence of hadronic matter at thermodynamical equilibrium also has important consequences in other areas: such matter was possibly the first produced during the evolution of the early universe. It is also conjectured that the effects of such a system in neutron stars has strong implications for the stability of those objects. In this work, a brief review on the idea of fireball and on the description of its thermodynamical properties is presented. It is shown that the self-similarity of hadronic systems can be a relevant aspect of those properties, and that Tsallis statistics can be the best framework for its description.

One of the first and most fruitful attempts to describe the thermodynamics of fireballs was the one advanced by Rolf Hagedorn [2]. The crucial point in his theory was evident already in the very definition of fireballs, which was proposed by Hagedorn as: fireball is $(*)$ a statistically equilibrated system formed by an undetermined number of fireballs, each of them in turn being (goto *). This is an unusual definition for a physical system, since it is recursive: the word fireball appears in its own definition 
and it could therefore be regarded as useless. However, Hagedorn succeded in obtaining the entire thermodynamics of fireballs based essentially on that definition, as is shown below.

\section{Hagedorn's Self-Consistence Principle}

Since fireballs are made of fireballs, Hagedorn conceived an ideal gas of such systems, which should be itself a fireball. Then, the partition function can be written as [2],

$$
\begin{aligned}
\log \left[1+Z\left(V_{o}, T\right)\right] & =\frac{V_{o}}{2 \pi^{2}} \int_{0}^{\infty} d m \int_{0}^{\infty} d p p^{2} \times \\
& {\left[\rho_{F}(m) \log \left[1+\exp \left(-\beta \sqrt{p^{2}+m^{2}}\right)-\rho_{B}(m) \log \left[1-\exp \left(-\beta \sqrt{p^{2}+m^{2}}\right)\right]\right],\right.}
\end{aligned}
$$

where $\beta=1 / T$, and $\rho_{F(B)}(m)$ is the mass spectrum of fermions (bosons) in the gas. After Taylor expansion of the logarithm at the right-hand side, one obtains

$$
\log \left[1+Z\left(V_{o}, T\right)\right]=\frac{V_{o}}{2 \pi^{2}} \sum_{n=1}^{\infty} \frac{1}{n} \int_{0}^{\infty} d m \int_{0}^{\infty} d p p^{2} \rho(n ; m) \exp \left\{-n \beta \sqrt{p^{2}+m^{2}}\right\},
$$

where

$$
\rho(n ; m)=\rho_{B}(m)-(-1)^{n} \rho_{F}(m),
$$

$V_{o}$ is the gas volume, and for energies high enough only the term corresponding to $n=1$ is relevant. In this case, $\rho(n=1 ; m)=\rho(m)$ is the hadron mass spectrum of a gas composed of fermions and bosons, and it corresponds to the sum of the fermions and bosons mass spectra given above.

As it happens for any system, the partition function can also be written in terms of the density of states, $\sigma(E)$, as

$$
Z\left(V_{0}, T\right)=\int_{0}^{\infty} \sigma(E) \exp \{-\beta E\} d E .
$$

Obviously, one must necessarily have

$$
Z_{1}=Z_{2}
$$

where $Z_{1}$ and $Z_{2}$ are the two forms of partition function in Equations (2) and (4), respectively.

In general, $\rho(m)$ and $\sigma(E)$ are distinct functions, but in the case of fireballs, since the internal energy of the gas can be related, they must be related to one another. In fact, Hagedorn imposes the so-called weak-constraint

$$
\log [\rho(m)]=\log [\sigma(E)],
$$

meaning that both functions must be of the same order of magnitude. In addition, he considered that such equality should hold only asymptotically.

Most of the work performed by Hagedorn, as described in Ref. [2], was to look for function $\rho(m)$ and $\sigma(E)$ that simultaneously satisfy Equations (5) and (6). It results that such functions exist and have the form

$$
\rho(m)=m^{-5 / 2} \exp \left[m / k T_{0}\right]
$$

and

$$
\sigma(E)=E^{a} \exp \left[E / k T_{o}\right],
$$

where $T_{o}$ and $a$ are constant parameters. With these functions, both forms of the partition function reduce to

$$
Z(T) \sim\left[\frac{1}{\beta-\beta_{o}}\right]^{a+1}
$$

where $\beta=1 / T$ and $\beta_{o}=1 / T_{o}$ and $a+1=\gamma V_{o} /\left(2 \pi^{2} \beta^{3 / 2}\right)$, and $\gamma$ is a constant. 
These are important results of the Hagedorn's theory that can be directly verified experimentally. The energy distribution of the particles in the fireball, or equivalently the transverse momentum $\left(p_{T}\right)$ distribution, which is easier accessed in experiments, is given by

$$
\frac{d^{2} N}{d p_{T} d y}(y=0)=\frac{g V}{(2 \pi)^{2}} p_{T} m_{T} \exp \left(\frac{m_{T}-\mu}{T}\right)
$$

where $m_{T}=\sqrt{p_{T}^{2}+m^{2}}$ and $\mu$ is the chemical potential, so by fitting such formula to data the temperature, $T$, is obtained. It was verified that for collisions at sufficiently high energies the temperature of the formed fireball is independent of the collision energy of particle species, in accordance with the theoretical predictions.

The mass spectrum in Equation (7) can be fitted to the observed number of hadronic states as a function of mass, obtaining the parameter $T_{0}$. It was found that the the formula for $\rho(m)$ indeed describes the observed mass spectrum above $\sim 800 \mathrm{MeV}$, and $T_{0}$ can be obtained. It was fundamental for the success of Hagedorn theory that the limiting value of temperature found in $p_{T}$ distribution analyses was equal to the parameter $T_{o}$. Thus, the predictions from Hagedorn's theory were fully supported by data.

This success prompted many works in the following years: Frautshi [3], with his bootstrap model where hadrons are supposed to be made of hadrons - as is the case for fireballs_-obtained the same mass spectrum formula firstly obtained by Hagedorn. This result shows the strict connection between structure and thermodynamics in the case of hadrons. Hadron resonance gas (HRG) models were formulated [4], and the theoretical basis for the adoption of an ideal gas model to describe a fireball was put forward, based on Feynman-Dyson expansion [5].

The theoretical framework for the thermodynamics of fireballs was completely established, and the Hagedorn temperature, $T_{0}$, became a matter of fact. Based on the MIT bag model for hadrons, Cabibbo and Parisi [6] interpreted $T_{o}$ as a critical temperature for the phase transition between the known confined regime of quarks and gluons to a hypothesized deconfined regime, linking Hagedorn's theory to the recently developed quark structure of hadrons. This work triggered the search for the quark-gluon plasma-a new state of matter whose existence was proved experimentally only after three decades of intense efforts.

Despite the astonishing success in predicting so many characteristics of the hot and dense hadronic matter, as the beam energy increased and a larger range of $p_{T}$ distribution became available, Hagedorn's theory was found to fail in completely describing the outcome of HECs. In fact, Hagedorn himself proposed an alternative empirical model [7] in substitution to his thermodynamical theory. A natural question that arises is: what went wrong? To answer this question, a special kind of fractal structure is discussed in the next section. In what follows, only the hadronic matter is considered, so a phase transition to the quark-gluon plasma cannot be analysed.

\section{The Fractal Structure of Hadrons}

The essential aspect of both Hagedorn's definition for fireballs and Frautshi's definition for hadrons is the self-similarity present in their definitions. Self-similar structures were more deeply investigated only two decades after Hagedorn proposed his thermodynamics, when Mandelbrot [8] introduced the concept of fractals (i.e., systems with fractional dimensions). Experimental evidence of a fractal structure in multiparticle production in HEC were first noticed through the study of intermittence in high-energy distributions $[9,10]$. The fractal dimension obtained in such analyses was found to be in the range $D=0.45$ to 0.65 for $p p$ collisions and $e^{+} e^{-}$collisions [11-19]. The origin of this fractal dimension was attributed to the dynamical process of multiparticle production [20-22]. The fractal dimension obtained in intermittence analysis from $p p$ collisions is in the range 0.43 to 0.65 [23-27]. Such analyses are rather complex and delicate, with many factors that can disturb the 
results $[28,29]$. The limitations of this approach were already recognized by the beginning of the new millenium.

Further evidence for self-similarity is the z-scaling [30,31]. High-energy distributions were shown to scale with the variable $z=z_{0} \Omega^{-1}$, where

$$
\Omega=\left(1-x_{1}\right)^{\delta_{1}}\left(1-x_{2}\right)^{\delta_{2}}\left(1-y_{1}\right)^{\epsilon_{1}}\left(1-y_{2}\right)^{\epsilon_{2}},
$$

with $x_{1}$ and $x_{2}$ being the fraction of momentum carried by the initial colliding partons and $\epsilon_{1}$ and $\epsilon_{2}$ being the faction of momentum carried by the outgoing partons. The exponents in the expression above represent the fractal dimensions of the phase-space.

Expressed in therms of such $z$ variable, all data fall along one single function $\psi(z)$ given by

$$
\psi(z)=\frac{\pi}{(d N / d y) \sigma_{\text {in }}} J^{-1} \frac{d^{3} \sigma}{d^{3} p}
$$

where $J$ is the Jacobian of the transformation $\left(p_{T}, y\right) \rightarrow(z, \eta)$, and $d N / d y$ is the central differential multiplicity with respect to the rapidity $y$. The fractal dimension that results from the $z$-scaling is $D=0.5$, in nice agreement with the results from intermittence analysis.

Self-symmetry in high-energy collisions was recently proved in a very direct way. Wilk and Wlodarcyk [32] have shown that jets produced in those collisions present the same $p_{T}$ distribution followed by single hadrons, which is strong evidence that a jet is a fireball as much as hadrons are. Moreover, when the distribution of the transversal momentum of the jet particles with respect to the direction of the jet is calculated, its distribution is also similar to the distribution of hadrons and jets with respect to the beam direction. This means that not only are jets fireballs, but the particles inside the jets are also fireballs.

Only recently has a connection between the experimental evidence of self-similarity in HEC and the Hagedorn and Frautschi models for fireball and hadrons been attempted $[33,34]$ by introducing the concept of thermofractals; that is, systems with fractal structure in their thermodynamical quantities. It turns out that with such structure, Hagedorn's self-consistence principle can be rescued from its apparent failure, as discussed below.

\subsection{Thermofractals}

Thermofractals are defined as systems in thermodynamical equilibrium presenting the following three properties [33,34]:

1. The total energy is given by

$$
U=F+E,
$$

where $F$ corresponds to the kinetic energy of $N^{\prime}$ constituent subsystems and $E$ corresponds to the internal energy of those subsystems, which behaves as particles with an internal structure.

2. The constituent particles are thermofractals. The ratio $\langle E\rangle /\langle F\rangle$ is constant for all the subsystems. However, the ratio $E / F$ can vary according to a distribution which is self-similar, $\tilde{P}(E)$; that is, at different levels of the subsystem hierarchy the distribution of the internal energy is equal to that in the other levels.

3. At some level $n$ in the hierarchy of subsystems, the phase space is so narrow that one can consider

$$
\tilde{P}\left(E_{n}\right) d E_{n}=\rho d E_{n},
$$

with $\rho$ being independent of the energy $E_{n}$.

The energy distribution of a thermodynamical system is given according to Boltzmann statistics by

$$
P(U) d U=A \exp (-U / k T) d \Gamma,
$$


where $d \Gamma$ is an infinitesimal volume in the phase space. In the case of thermofractals, the phase space must include momentum degrees of freedom of free particles as well as the internal degrees of freedom; therefore, one can write

$$
d \Gamma=V d^{3 N^{\prime}} p d E
$$

where $p$ is a momentum component for one of the particles composing the gas, while $d E$ depends on their internal degrees of freedom and V is the volume of the gas. Since kinetic and internal degrees of freedom are independent, one can integrate in the momentum coordinates, obtaining the well-known result

$$
\int_{-\infty}^{\infty} \exp \left(-\sum_{i=1}^{3 N^{\prime}} \frac{p_{i}^{2}}{2 m k T}\right) d^{3} p \propto \int_{0}^{\infty} F^{3 N^{\prime} / 2} \exp \left(\frac{F}{k T}\right) d F,
$$

where $F$ is the kinetic energy of the $N^{\prime}$ compound thermofractals.

For the internal degrees of freedom, according to property 2 of self-similar thermofractals [33], the part related to internal degrees of freedom is given by

$$
d E=\frac{F}{k T}[\tilde{P}(\varepsilon)]^{v} d \varepsilon
$$

with $v$ an exponent that will be determined below, and

$$
\frac{\varepsilon}{k T}=\frac{E}{F}
$$

Defining

$$
\alpha(\varepsilon)=1+\frac{\varepsilon}{k T}
$$

Equation (15) is now given by

$$
P(U) d U=A F^{\frac{3 N}{2}-1} \exp \left(-\frac{\alpha F}{k T}\right) d F \tilde{P}(\varepsilon) d \varepsilon,
$$

where $N=N^{\prime}+2 / 3$ is an effective number of particles that accounts for the relevant internal degrees of freedom and $A$ is a normalization constant.

The thermodynamical potential is given by

$$
\Omega=\int_{0}^{\infty} A F^{\frac{3 N}{2}-1} \exp \left(-\frac{\alpha F}{k T}\right) d F[\tilde{P}(\varepsilon)]^{v} d \varepsilon .
$$

which after integration on $F$ results in

$$
\Omega=\int_{0}^{\infty} A\left[1+\frac{\varepsilon}{k T}\right]^{3 N / 2}[\tilde{P}(\varepsilon)]^{v} d \varepsilon .
$$

It is possible to impose the identity

$$
P(U)=\tilde{P}(\varepsilon)
$$

corresponding to a self-similar solution for the thermofractal probability distributions. The simultaneous solution for Equations (23) and (24) is obtained with

$$
P(\varepsilon)=A\left[1+\frac{\varepsilon}{k T}\right]^{-\frac{3 N}{2} \frac{1}{1-v}} .
$$


Defining the quantities $q$ and $\tau$ by

$$
q-1=\frac{2}{3 N}(1-v)
$$

and

$$
\tau=\frac{2(1-v)}{3} T
$$

it results from Equation (25)

$$
P(\varepsilon)=A e_{q}(-\varepsilon / \tau)
$$

where

$$
e_{q}(x)=[1+(1-q) x]^{\frac{1}{1-q}}
$$

is the q-exponential function. Equation (28) is exactly the Tsallis q-exponential factor characteristic of the nonextensive statistics [35].

The fact that the probability distribution for thermofractals is the Tsallis q-exponential has several consequences. It establishes a new connection between Boltzmann statistics and Tsallis statistics, aside from the ones already known based on Fokker-Planck equation [36], on fluctuation temperature [37-39], and on finite size of the system [40]. However, this time it is not based in thermodynamical properties, but on the the system structure, allowing a direct connection with energy fluctuation. As usual, the limit $q \rightarrow 1$ reduces to the Boltzmann statistics, and from Equations (26) and (27) it is possible to see that this limit corresponds to $\tau<<T$; therefore, the internal energy, $E$, is much smaller than the kinetic energy, $F$, which corresponds to an ideal gas in the Boltzmann statistics. Furthermore, Equation (28) shows that the use of the q-exponential factor-instead of the exponential one that appears in Boltzmann statistics-allows a direct application of Thermodynamics rules to calculate all necessary quantities without worrying about the complexities of the thermofractal structure.

With the results obtained above it is now possible to understand what went wrong with Hagedorn's theory for fireballs, which was the question posed in the Introduction. His own definition for the system was already indicating that it presents a fractal structure, and a system with fractal structure is described by Tsallis statistics, not by Boltzmann statistics, in the sense discussed above. In this regard, it is interesting to mention that Fowler and Weiner [41] had already noticed that a fireball as proposed by Hagendorn could not present a phase transition if the exponential distribution was the relevant characteristic of its thermodynamical description, but rather a power-law distribution would be necessary. The result obtained here is therefore in agreement with that prediction. The problem now is to find out if the self-consistence principle has a solution when formulated in terms of Tsallis statistics. In Ref. [42] it was shown that Hagedorn's principle can indeed be generalized to a non-extensive version, as discussed below.

\subsection{Non-Extensive Self-Consistent Thermodynamics}

In order to extend Hagedorn's theory with non-extensive statistics, it is necessary to know the partition function of an ideal gas in that statistics. Such a function was determined in Ref. [43] and presents a form that is very similar to the classical partition function for an ideal gas in Boltzmann statistics, resulting that the only difference in the partition function is the use of the q-exponential factor instead of the exponential one. The generalized version of the partition functions (see Equations (1) and (4)) results in

$$
\log \left[1+Z_{q}\left(V_{o}, T\right)\right]=\frac{V_{o}}{2 \pi^{2}} \sum_{n=1}^{\infty} \frac{1}{n} \int_{0}^{\infty} d m \int_{0}^{\infty} d p p^{2} \rho(n ; m)\left[1+(q-1) \beta{\sqrt{p^{2}+m^{2}}}^{-\frac{n q}{(q-1)}},\right.
$$


and

$$
Z_{q}\left(V_{o}, T\right)=\int_{0}^{\infty} \sigma(E)[1+(q-1) \beta E]^{-\frac{q}{(q-1)}} d E
$$

It was shown [42] that the self-consistency can be asymptotically achieved by choosing

$$
\rho(m)=\frac{\gamma}{m^{5 / 2}}\left[1+\left(q_{o}-1\right) \beta_{o} m\right]^{\frac{1}{q_{o}-1}}
$$

and

$$
\sigma(E)=b E^{a}\left[1+\left(q_{o}-1\right) E\right]^{\frac{1}{q_{o}-1}},
$$

where $\gamma$ is a constant. Here, $a, b$ and $\gamma$ are arbitrary constants. Note that for $q \rightarrow 1$ the two expressions above approach the corresponding expressions in Hagedorn's theory.

The partition function results in

$$
Z_{q}\left(V_{o}, T\right) \rightarrow b \Gamma(a+1)\left(\frac{1}{\beta-\beta_{o}}\right)^{a+1} .
$$

with

$$
a+1=\frac{\gamma V_{o}}{2 \pi^{2} \beta^{3 / 2}} .
$$

Thus, when Hagedorn's principle is extended by using the non-extensive statistics, a non-extensive self-consistent thermodynamics (NESCT) is obtained It is found that the limiting (or critical) temperature is still obtained, while a new constant-the entropic index $q$-characterizes all hadrons. In addition, new formulas for the mass spectrum and for the density of states of hadrons are obtained. The new density of states leads to a transverse momentum distribution given by

$$
\frac{d^{2} N}{d p_{T} d y}(y=0)=\frac{g V}{(2 \pi)^{2}} p_{T} m_{T}\left[1+(q-1) \frac{m_{T}-\mu}{T}\right]^{-q /(q-1)},
$$

which has been used in many studies to describe the experimental data. All the results approach Hagedorn's results as $q \rightarrow 1$. The resulting values for $q$ and $T$ support the above findings of a constant temperature and entropic index, regardless of the collision energy (as long as it is above approximately $1 \mathrm{TeV}$ ) or the particle species [44-57].

The new mass spectrum formula was used to describe the known hadronic states up to mass $m=2 \mathrm{GeV}[53,54]$, resulting in values for $T$ and $q$ that are in good agreement with the results from $p_{T}$ distribution analyses, thus showing that the predictions from the NESCT are fully supported by experiments. In fact, the new mass spectrum formula can describe the observed hadronic states even better than Hagedorn's formula, showing good agreement with data even for masses as small as that for pions.

From the values for $T$ and $q$ obtained from mass spectrum analysis or from $p_{T}$ distribution analysis, it is possible to obtain all thermodynamics functions, and in Ref. [58] it was shown that the results obtained are in good agreement with lattice QCD data. All these results shows that the assumption of a fractal structure of hadrons is consistent with the experimental information available. Indeed, the fractal dimension can be calculated using the values for $T$ and $q$, and results in $D=0.45$, which is in good agreement with the results from intermittence analysis and from z-scaling.

\section{Discussion and Conclusions}

There are many works applying the extended Hagedorn distribution to fit experimental data (see Refs. [50,59-64] for recent works on the subject); nevertheless, the debate around the thermodynamical properties of the fireball formed at high energies continues. The theory exposed here shows that different concepts such as fireballs, intermittence, hadron structure, self-similarity, thermofractal 
structure, and self consistence principle are braided into one single theoretical framework with only two parameters.

In the theoretical approach proposed here, the fractal dimension observed in high-energy data is explained through the thermofractal parameter, $q$, that is also the entropic index in Tsallis statistics. It is shown that the best statistics to describe fireball thermodynamics is Tsallis statistics-Boltzmann statistics being possible to be used only if all the complex structure of thermofractals are taken into account. The non-extensive self-consistent thermodynamics that results from the extension of Hagedorn's theory with Tsallis statistics is shown to give a very good description of HEC data in the whole range of $p_{T}$ distribution available from experiments. Additionally, the modified mass spectrum formula describes the observed hadron states very well, even for states with masses as low as pion mass. Additionally, the thermofractal structure allows for a new interpretation of the generalization to Boltzmann statistics provided by Tsallis entropic form.

This theory also allows for further developments. Clearly, the thermofractal structure proposed here for hadrons should present itself in parton distribution functions (PDFs), and it is worth noticing that a PDF inspired in fractal structure was already proposed several years ago [65], showing good agreement with data. To this end, one further step must be accomplished by considering that the energy distribution considered here is related to quantum fluctuations of the hadron energy. In this case, one can conjecture that the parameter $q$ and the related fractal dimension may be connected to the running constant that regulates QCD interaction.

The subject presented here is still under debate. Some analyses of experimental data suggest that the parameters $q$ and $T$ are not constant (as demanded by theory), but depend on collision energy or particle species and also on multiplicity [66,67], even at energies as high as $13 \mathrm{TeV}$. Although some dependence on all these parameters is possible at low energies, a variation for high energies was not expected. However, it should be emphasized that the $p_{T}$ analysis is complex, and the fact that the parameters $q$ and $T$ are correlated (as described above) contributes to variations in the results if such correlations are not carefully taken into account. In addition, high statistics is necessary in a large $p_{T}$ range in order to extract precise information from data. Applications of such theory have already been made in astrophysics, where the stability of neutron stars has been studied under the light of the non-extensive thermodynamics [68], showing effects that help to understand recent observation of more massive objects of this kind. Recently, the MIT Bag Model has been extended to include the thermofractal structure described here by introducing the non-extensive distributions [69].

Acknowledgments: This work was supported by Conselho Nacional de Pesquisa (CNPq) under grant 310982/2014-6.

Conflicts of Interest: The authors declare no conflict of interest.

\section{References}

1. Fermi, E. High energy nuclear events. Prog. Theor. Phys. 1950, 5, 570-583.

2. Hagedorn, R. Statistical thermodynamics of strong interactions at high energies. Nuovo Cim. Suppl. 1965, 3, 147-186.

3. Frautschi, S. Statistical bootstrap model of hadrons. Phys. Rev. D 1971, 3, 2821-2833.

4. Venugopalan, R.; Prakash, M. Thermal properties of interacting hadrons. Nucl. Phys. A 1992, 546, 718-760.

5. Dashen, R.; Ma, S.; Bernstein, H.J. S-matrix formulation of statistical mechanics. Phys. Rev. 1969, 187, 345-370.

6. Cabibbo, N.; Parisi, G. Exponential hadronic spectrum and quark liberation. Phys. Lett. B 1975, 59, 67-69.

7. Hagedorn, R. Multiplicities, $p_{T}$-distribution and the expected hadron $\rightarrow$ quark-gluon phase transition. La Rivista del Nuovo Cimento 1983, 6, 1-50.

8. Mandelbrot, B.B. The Fractal Geometry of Nature; WH Freeman: New York, NY, USA, 1983.

9. Bialas, A.; Peschanki, R. Moments of rapidity distributions as a measure of short-range fluctuations in high-energy collisions. Nucl. Phys. B 1986, 273, 703-718. 
10. Bialas, A.; Peschanki, R. Intermittency in multiparticle production at high energy. Nucl. Phys. B 1988, 308, 857-867.

11. Hwa, R.C. Fractal measures in multiparticle production. Phys. Rev. D 1990, 41, 1456-1462.

12. Hwa, R.C.; Pan, J. Fractal behavior of multiplicity fluctuations in high-energy collisions. Phys. Rev. D 1992, 45, 1476-1483.

13. Hegyi, S. Monofractal density fluctuations and scaling laws for count probabilities and combinants. Phys. Lett. B 1993, 318, 642-647.

14. Dremin, I.M.; Hwa, R.C. Quark and gluon jets in QCD: Factorial and cumulant moments. Phys. Rev. D 1994, $49,5805-5811$.

15. Dremin, I.M.; Levtchenko, B.B. Fractal 'hot spots' and structure functions at low $x_{B j}$. AIP Conf. Proc. 1993, 276, 454-458.

16. Hegyi, S.; Csörgö, T. On the intermittency signature of quark-gluon plasma formation. Phys. Lett. B 1992, 296, 256-260.

17. Xie, Y.L.; Chen, G.; Wang, J.L.; Liu, Z.H.; Wang, M.J. Scaling properties of multiplicity fluctuations in heavy-ion collisions simulated by AMPT model. Nucl. Phys. A 2013, 920, 33-44.

18. Kittel, W.; de Wolf, E.A. Soft Multihadron Dynamics; World Scientific: Singapore, 2005.

19. De Wolf, E.A.; Dremin, I.M.; Kittel, W. Scaling laws for density correlations and fluctuations in multiparticle dynamics. Phys. Rep. 1996, 270,1-141.

20. Brax, P.; Peschanski, R. Multifractal analysis of intermittency and phase transitions in multiparticle dynamics. Nucl. Phys. B 1990, 346, 65-83.

21. Brax, P.; Peschanski, R. Intermittency and hidden phase-transition in multiparticle collisions. Nucl. Phys. B 1991, 353, 165-182.

22. Bialas, A.; Zalewski, K. Phase structure of self-similar multiparticle systems and experimental determination of intermittency parameters. Phys. Lett. B 1990, 238, 413-416.

23. Ajienko, I.V.; Belokopytov, Y.A.; Böttcher, H.; Botterweck, F.; Chliapnikov, P.V.; Crijns, F.; Garutchava, Z.G. Intermittency patterns in $\pi+\mathrm{p}$ and $\mathrm{K}+\mathrm{p}$ collisions at $250 \mathrm{GeV} /$ c. Phys. Lett. B 1989, 222, 306-310.

24. Rasool, M.H.; Ahmad, M.A.; Ahamad, S. Multifractal study and multifractal specific heat of singly charged particles produced in 32 S-Em interactions at 200 AGeV. Chaos Solitons Fractals 2015, 81, 197-202.

25. Singh, G.; Jain, P.L. Multifractal analysis of emulsion 197 collisions at 10.6A GeV. Phys. Rev. C 1994, 50, 2508-2515.

26. Albajar, C.; Albajar, C.; Allkofer, O.C.; Apsimon, R.; Bartha, S.; Bezaguet, A.; Boehrer, A.; Coughlan, J.A. Multifractal analysis of minimum bias events in $\sqrt{s}=630 \mathrm{GeV} \bar{p} p$ collisions. Z. Phys. C Part. Field 1992, 56, 37-46.

27. Ghosh, D.; Deb, A.; Chattopadhyay, R.; Sarkar, S.; Jafry, A.K.; Lahiri, M.; Das, S. Evidence of multifractal nature of target-evaporated slow particles produced in ultrarelativistic heavy ion interactions. Phys. Rev. C 1998, 58, 3553-3559.

28. Sarkisyan, E.K.G. Description of local multiplicity fluctuations and genuine multiparticle correlations. Phys. Lett. B 2000, 477, 1-12.

29. Lipa, P.; Buschbeck, B. From strong to weak intermittency. Phys. Lett. B 1989, 223, 465-469.

30. Tokarev, M.; Zborovsky, I. Top-Quark pT-Spectra at LHC and Flavor Independence of z-Scaling. In Proceedings of the XLVI International Symposium on Multiparticle Dynamics (ISMD2016), Jeju island, Korea, 29 August-2 September 2016; Volume 141, pp. 02006.

31. Zborovský, I.; Tokarev, M.V. Generalized z-scaling in proton-proton collisions at high energies. Phys. Rev. D 2007, 75, 094008.

32. Wilk, G.; Włodarczyk, Z. Self-similarity in jet events following from pp collisions at LHC. Phys. Lett. B 2013, 727, 163-167.

33. Deppman, A. Thermodynamics with fractal structure, Tsallis statistics, and hadrons. Phys. Rev. D 2016, 93, 054001.

34. Deppman, A.; Megías, E. Fractal aspects of hadrons. In Proceedings of the XLVI International Symposium on Multiparticle Dynamics (ISMD2016), Jeju island, Korea, 29 August-2 September 2016; Volume 141, pp. 01011.

35. Tsallis, C. Possible generalization of Boltzmann-Gibbs statistics. J. Stat. Phys. 1988, 52, 479-487.

36. Borland, L. Ito-Langevin equations within generalized thermostatistics. Phys. Lett. A 1998, 245, 67-72.

37. Beck, C. Generalized statistical mechanics of cosmic rays. Phys. A Stat. Mech. Appl. 2004, 331, 173-181. 
38. Wilk, G.; Wlodarczyk, Z. Multiplicity fluctuations due to the temperature fluctuations in high-energy nuclear collisions. Phys. Rev. C 2009, 79, 054903.

39. Wilk, G. Fluctuations, correlations and the nonextensivity. Braz. J. Phys. 2007, 37, 714-716.

40. Biró, T.; Barnaföldi, G.G.; Ván, P. Quark-gluon plasma connected to finite heat bath. Eur. Phys. J. A 2013, $49,110$.

41. Fowler, G.N.; Weiner, R.M. Incompatibility of an exponential mass spectrum with the existence of a quark phase. Phys. Lett. B 1980, 89, 394-396.

42. Deppman, A. Self-consistency in non-extensive thermodynamics of highly excited hadronic states. Phys. A Stat. Mech. Appl. 2012, 391, 6380-6385.

43. Megías, E.; Menezes, D.P.; Deppman, A. Non extensive thermodynamics for hadronic matter with finite chemical potentials. Phys. A Stat. Mech. Appl. 2015, 421, 15-24.

44. Bediaga, I.; Curado, E.M.F.; de Miranda, J.M. A nonextensive thermodynamical equilibrium approach in $\mathrm{e}^{+} \mathrm{e}^{-} \rightarrow$ hadrons. Phys. A Stat. Mech. Appl. 2000, 286, 156-163.

45. Beck, C. Non-extensive statistical mechanics and particle spectra in elementary interactions. Phys. A Stat. Mech. Appl. 2000, 286, 164-180.

46. Sena, I.; Deppman, A. Systematic analysis of pT-distributions in p+ p collisions. Eur. Phys. J. A 2013, 49, 17.

47. Cleymans, J.; Worku, D.J. The Tsallis distribution in proton-proton collisions at $=0.9 \mathrm{TeV}$ at the LHC. Phys. G Nucl. Part. Phys. 2012, 39, 025006.

48. Azmi, M.D.; Cleymans, J. Transverse momentum distributions in proton-proton collisions at LHC energies and Tsallis thermodynamics. J. Phys. G 2014, 41, 065001.

49. Azmi, M.D.; Cleymans, J. The Tsallis distribution at large transverse momenta. Eur. Phys. J. C 2015, 75, 430.

50. Wong, C.-Y.; Wilk, G.; Cirto, L.; Tsallis, C. From QCD-based hard-scattering to nonextensive statistical mechanical descriptions of transverse momentum spectra in high-energy pp and pp collisions. Phys. Rev. D 2015, 91, 114027.

51. Wong, C.-Y.; Wilk, G. Tsallis fits to p T spectra and multiple hard scattering in pp collisions at the LHC. Phys. Rev. D 2013, 87, 114007.

52. Rybczyński, M.; Włodarczyk, Z.; Wilk, G. On the possibility of $q$-scaling in high-energy production processes. J. Phys. G Nucl. Part. Phys. 2012 39, 095004.

53. Marques, L.; Andrade-II, E.; Deppman, A. Nonextensivity of hadronic systems. Phys. Rev. D 2013, 87, 114022.

54. Marques, L.; Cleymans, J.; Deppman, A. Description of high-energy pp collisions using Tsallis thermodynamics: Transverse momentum and rapidity distributions. Phys. Rev. D 2015, 91, 054025.

55. Abelev, B.; Adam, J.; Adamova, D.; Adare, A.M.; Aggarwal, M.M.; Rinella, G.A.; Masoodi, A.A. Production of $K^{*}(892)^{0}$ and $\phi(1020)$ in pp collisions at $\sqrt{s}=7$ TeV. Eur. Phys. J. C 2012, 72, 2183.

56. Khachatryan, V.; Sirunyan, A.M.; Tumasyan, A.; Adam, W.; Bergauer, T.; Dragicevic, M.; Erö, J.; Fabjan, C.; Friedl, M.; Frühwirth, R. Strange particle production in pp collisions at $\sqrt{s}=0.9$ and 7 TeV. J. High Energy Phys. 2011, 2011, 64 .

57. Self-affine fractality in $\pi^{+} \mathrm{p}$ and $K^{+} \mathrm{p}$ collisions at $250 \mathrm{GeV} /$ c. Phys. Lett. B 1996, 382, 305-311.

58. Deppman, A. Properties of hadronic systems according to the nonextensive self-consistent thermodynamics. J. Phys. G 2014, 41, 055108.

59. Kiess, T.E. A resolution of a metric singularity associated with the introduction of $\Lambda$ into static spherically symmetric systems. Int. J. Mod. Phys. D 2017, 26, 1750039.

60. Mizoguchi, T.; Biyajima, M.; Suzuki, N.; Analyses of whole transverse momentum distributions in $p p$ and $p p$ collisions by using a modified version of Hagedorn's formula. Int. J. Mod. Phys. A 2017 32, 1750057.

61. Parvan, A.S. Ultrarelativistic transverse momentum distribution of the Tsallis statistics. Eur. Phys. J. A 2017, 53.

62. Zheng, H.; Bonasera, A. Systematic analysis of hadron spectra in $p+p$ collisions using Tsallis distributions. Phys. Rev. D 2015, 92, 074009.

63. Biro, G.; Barnafoldi, G.G.; Biro, T.S.; Urmossy, K.;Takacs, A. Systematic analysis of the non-extensive statistical approach in high energy particle collisions-Experiment vs. theory. Entropy 2017, 19, 88.

64. Li, B.; Bai, T.; Guo, Y.; Liu, F.H. On J/ $\psi$ and Y Transverse Momentum Distributions in High Energy Collisions. Adv. High Energy Phys. 2017, 2017, 9383540.

65. Lastovicka, T. Self-similar Properties of the Proton Structure at low x. Eur. Phys. J. C Part. Fields 2002, 24, 529-533. 
66. Khuntia, A.; Tripathy, S.; Sahoo, R.; Cleymans, J. Multiplicity dependence of non-extensive parameters for strange and multi-strange particles in proton-proton collisions at $\sqrt{s}=7 \mathrm{TeV}$ at the LHC. Eur. Phys. J. A 2017 53, 103 .

67. Grigoryan, S. Using the Tsallis distribution for hadron spectra in pp collisions: Pions and quarkonia at $\sqrt{s}=5-13,000$ GeV. Phys. Rev. D 2017, 95, 056021.

68. Menezes, D.P.; Deppman, A.; Megias, E.; Castro, L.B. Non-extensive thermodynamics and neutron star properties. Eur. Phys. J. A 2015, 51, 155.

69. Cardoso, P.H.G.; da Silva, T.N.; Deppman, A.; Menezes, D.P. Quark matter revisited with non extensive MIT bag model. arXiv 2017, arXiv:1706.02183.

(C) 2017 by the authors. Licensee MDPI, Basel, Switzerland. This article is an open access article distributed under the terms and conditions of the Creative Commons Attribution (CC BY) license (http://creativecommons.org/licenses/by/4.0/). 